\title{
La Investigación Acción como herramienta para Formación Docente. Experiencia en la Carrera Ciencias Sociales de la Facultad de Ciencias de la Educación de la UNAN-Managua, Nicaragua
}

\author{
Julio César Orozco Alvarado ${ }^{1}$
}

\section{RESUMEN}

El presente artículo es el resultado de una investigación documental realizada en la carrera Ciencias Sociales sobre el número de graduados por año, también se realizó una investigación en el Centro de Documentación de la Facultad de Educación e Idiomas para conocer los tipos de investigaciones que han realizado los graduados y los resultados de una sistematización de las investigaciones realizadas en la Carrera. A partir de las técnicas y acciones antes mencionadas se llegó a la conclusión que la carrera está llevando a cabo la Misión de la Universidad y de la Facultad de Educación, la cual es formar profesionales y técnicos integrales. La Misión se ha venido logrando al hacer uso del paradigma investigativo Sociocrítico y la Investigación Acción como método de investigación. Este tipo de investigaciones se ha venido desarrollando a partir de las necesidades educativas identificadas en los estudiantes de los Institutos de Educación Secundaria de nuestro país. A partir de esto, se diseñan Unidades Didácticas en las disciplinas sociales que se imparten en Educación Secundaria y se realizan intervenciones didácticas para incidir en la calidad de los aprendizajes. Los resultados encontrados en las investigaciones revisadas indican que la Investigación Acción es una herramienta efectiva para la formación del profesorado de Ciencias Sociales. Además, así lo expresan tanto los graduados de la carrera como los estudiantes de Educación Secundaria que han participado en las intervenciones didácticas realizadas por los graduados.

Palabras claves: Investigación Acción/ Innovación Pedagógica/ Intervención Didáctica

Recibido: 17 de agosto de 2016

Aceptado: 13 de octubre de 2016

1 Docente Titular Facultad de Educación e Idiomas, candidato a Doctor en Educación e Intervención Social por la Universidad Pablo de Olavide, España y UNAN- Managua. Correo electrónico: jorozcoa@hotmail.com 


\title{
Action Research as a tool for teacher training: Experience from the social sciences program of the Faculty of Education Sciences of UNAN-Managua, Nicaragua
}

\begin{abstract}
The present article derives from a documental research carried out from the Social Sciences program, on the number of graduates per year. Moreover, a project was conducted in which the different researches theses developed from the program were analyzed. The research concluded that the Social Sciences program supports the university's mission, which is to form integral professionals. This type of research has been conducted, due to the needs identified among students of Nicaraguan secondary schools. Hence, a set of didactic units were designed in order to make an incidence on the quality of the learnings. The research theses reveal that the Applied Research method is an effective tool to train Social Sciences teachers. Furthermore, the program's graduates that have participated in didactic interventions confirm the effectiveness of this research method.
\end{abstract}

Key words: Action research/Educational innovation/Didactic intervention. 


\section{INTRODUCCIÓN}

La Universidad Nacional Autónoma de Nicaragua, tiene como misión "formar profesionales y técnicos integrales desde y con una concepción científica y humanista del mundo, capaces de interpretar los fenómenos sociales y naturales con un sentido crítico, reflexivo y propositivo, para que contribuyan al desarrollo social" (Modelo Curricular UNAN- Managua, 2011, p. 19). En este mismo documento curricular nuestra institución se propone como objetivo estratégico "promover la innovación pedagógica mediante la investigación y sistematización del quehacer educativo, en los procesos de aprendizaje en cada uno de los niveles de formación que emprende la universidad...fortalecer la actitud creativa y emprendedora para el desarrollo de las investigaciones" (Modelo Curricular UNANManagua, 2011, p. 23), para dar cumplimiento a la Misión institucional la carrera Ciencias Sociales ha diseñado un plan de estudios que responda a las necesidades y problemáticas que enfrenta el Subsistema de Educación Básica y Media en los procesos de enseñanza-aprendizaje de las disciplinas sociales.

En este sentido la carrera Ciencias Sociales se ha propuesto como Misión formar docentes en el campo de las Ciencias Sociales con capacidad de abstracción, análisis, síntesis, comunicación oral y escrita, capaces de aprender a aprender y actualizarse permanentemente...el perfil laboral que caracteriza a esta carrera permite que el graduado se inserte como Docente y/o Jefe de área en el campo de las Ciencias Sociales, Promotor e Investigador Social y Educativo en escenarios urbanos y rurales, porque desarrollan la capacidad de investigar e identificar los problemas que obstaculizan el aprendizaje y el desarrollo social (Plan de Estudios Ciencias Sociales, 2013, p. 4).

Con base en la caracterización antes planteada, el Plan de Estudios de esta carrera se propone entre otros objetivos, los siguientes (Plan de estudios, 2013, pp. 16-17):
- Formar Profesores en el campo de las Ciencias Sociales, en las disciplinas de Geografía, Historia, Economía, Filosofía, Convivencia y Civismo, Sociología, Ética, Relaciones Humanas etc.

- Contribuir en la formación de Profesionales de la Educación a nivel de Licenciatura en el campo de las Ciencias Sociales con una concepción científica y humanista del mundo, capaces de interpretar los fenómenos sociales y naturales del mundo de hoy desde una perspectiva crítica y reflexiva de forma que contribuya al desarrollo socioeconómico del país.

- Incidir en los problemas relacionados con el aprendizaje y la enseñanza de los Estudios Sociales a través de la realización y ejecución de proyectos de intervención didáctica para desarrollar en los estudiantes aptitudes y actitudes positivas hacia las Ciencias Sociales.

- Investigar lo social y educativo bajo nuevos paradigmas universales, de forma que sean capaces de identificar los problemas que obstaculizan el aprendizaje de lo social y crear alternativas de solución para incidir en el desarrollo socioeconómico del país.

Como se puede observar existe una coherencia entre la Misión Institucional, la Misión de la Facultad de Educación e Idiomas y la Misión de la Carrera Ciencias Sociales, porque existe un compromiso y un encargo social como es formar profesionales y técnicos integrales en el campo de la Educación y de las Ciencias Sociales con las capacidades, actitudes y valores que luego pondrán en práctica en sus campos y ámbitos laborales.

\section{MATERIALES Y MÉTODOS}

El presente estudio es parte de una investigación realizada en la carrera Ciencias Sociales, Facultad de Educación e Idiomas, UNAN- Managua sobre una de las líneas de investigación que se lleva a cabo en esta carrera, ésta es la enseñanza-aprendizaje de las Ciencias 
Sociales, para ello se efectuó una revisión de las tesis realizadas por los graduados de la carrera Ciencias Sociales, esta revisión documental se realizó en el Centro de Documentación (CEDOC) de la Facultad de Educación e Idiomas. Luego esta investigación se complementó con una investigación bibliográfica sobre el paradigma Socio crítico y la Investigación Acción. Para finalizar la investigación, se realizó una consulta en el Sistema de Registro de Calificaciones (página web) de la UNAN- Managua, para extraer el número de graduados de los últimos cinco años en la carrera Ciencias Sociales. Lo que indica que la presente es una investigación documental de tipo exploratoria, basada en la práctica de los docentes investigadores de la Carrera Ciencias Sociales y la incidencia de este tipo de investigaciones en la práctica de los docentes del área de Ciencias Sociales en Educación Secundaria. Las principales técnicas utilizadas fueron la revisión documental, matriz de análisis de datos y la consulta de la página web de la Universidad (UNAN- Managua).

Con base en lo planteado en los párrafos que anteceden, se puede afirmar que esta es una investigación cualitativa de tipo exploratoria. Este tipo de estudios "se realizan cuando el objetivo es examinar un tema o problema de investigación poco estudiado, del cual se tienen muchas dudas o no se ha abordado antes" (Hernández, Fernández y Baptista, 2006, p. 100). Cabe destacar que este tipo de investigaciones son útiles porque "sirven para familiarizarnos con fenómenos relativamente desconocidos, obtener información sobre la posibilidad de llevar a cabo una investigación más completa...establecer prioridades para investigaciones futuras, o sugerir afirmaciones y postulados (Hernández, et al 2006, p. 101). Por los aspectos teóricos, metodológicos y por los hallazgos en el presente estudio, se puede afirmar que por sus características este es un estudio exploratorio, porque es una línea y método de trabajo poco practicado en nuestro país.

También esta investigación es de tipo descriptiva, este tipo de estudios" buscan especificar las propiedades, las características y los perfiles de personas, grupos, comunidades, procesos, objetos o cualquier otro fenómeno que se someta a un análisis...los estudios descriptivos únicamente pretenden medir o recoger información de manera independiente o conjunta sobre los conceptos o variables a las que se refieren" (Hernández, et al 2006, pp. 101-102). La presente es una investigación abierta y basada en una de las líneas de investigación de la carrera Ciencias Sociales de la Facultad de Educación e Idiomas y el objetivo es dar a conocer las bondades que tiene la aplicación de la Investigación Acción como método de investigación en la formación de docentes en las diferentes áreas del conocimiento, esto es, que este método de investigación se presta para aplicarse a las diferentes áreas del conocimiento. Es decir, que este tipo de intervenciones didácticas pueden realizarse tanto en las Ciencias Sociales como en las Ciencias Naturales.

\section{RESULTADOS Y DISCUSIÓN}

Los elementos planteados tanto en el Modelo Educativo de la Universidad como en el Plan de Estudios 2013 de la carrera Ciencias Sociales se debe a que en Nicaragua el desempeño profesional docente es un problema que ha afectado por años los subsistemas educativos, especialmente al subsistema de Educación Básica y Media, donde la calidad de los procesos de enseñanza-aprendizaje constantemente son puestos en tela de duda por el bajo desempeño laboral docente y el bajo rendimiento académico de los estudiantes que egresan de este subsistema.

De acuerdo con otros autores, Lucio 2014 considera en el desempeño laboral de los docentes están presentes estos paradigmas planteados producto de varios factores, uno es el empirismo de los docentes y otro es la resistencia al cambio por parte de algunos maestros de Educación Secundaria. Esta resistencia al cambio por parte de los maestros que ya están ubicados en Educación Secundaria afecta los docentes que se inician en la profesión docente, porque estos al insertarse laboralmente en el sistema educativo, asumen 
e interiorizan los modelos educativos tradicionalistas existentes en los centros educativos e institutos. Algunos docentes nóveles de este subsistema asumen estos modelos paradigmáticos por comodidad y otros por no entrar en contradicción con los docentes que por años han ejercido docencia en estos centros.

Con relación al párrafo anterior, López (2007) considera que "ningún modelo es mejor que otro, tan solo son diferentes y obedecen a momentos cronológicos". Lucio (2013) considera, que en el "desempeño laboral de los docentes confluyen dos paradigmas en pugna: Uno positivista cuantitativo, centrado en cuantificar y medir, desinteresado de los procesos de aprendizaje y la retroalimentación formativa; otro cualitativo, centrado en la persona en toda su complejidad, y en la integralidad de aspectos a evaluar en el aprendizaje, así como en procesos de retroalimentación y autorregulación." por eso considero que los docentes no debemos polarizar ninguna de las metodologías didácticas, ya que en la medida de lo posible podemos hacer una especie de eclecticismo en los procesos didácticos que llevamos a cabo de forma cotidiana.

Al desempeño profesional docente es necesario darle la importancia que este requiere, ya que del buen desempeño del maestro en el aula de clase dependen en gran medida los aprendizajes que adquieran los estudiantes. Lucio, (2014) considera que el desempeño docente depende de la formación de calidad que haya recibido el docente en la facultad donde se formó o se está formando. Él sugiere que los programas en donde se forman a los profesores deben propiciarles "modelos metodológicos innovadores y modelizadores, que inspiren a los maestros a transformar sus prácticas".

A los retos y desafíos planteados en la introducción , sobre la formación docente, uno de los factores que afecta a los subsistemas educativos, tanto al subsistema de Educación Básica y Media como a Educación Superior, que es donde ingresan los estudiantes que egresan de los Institutos de Educación
Secundaria, la carrera Ciencias Sociales ha adoptado la implementación de la Investigación Acción como método de investigación para la formación de los profesionales de la educación que se gradúan en la carrera, porque con este tipo de trabajos investigativos se lleva a cabo uno de los objetivos del Plan de Estudios de esta carrera, el cual es "incidir en los problemas relacionados con el aprendizaje y la enseñanza de los Estudios Sociales a través de la realización y ejecución de proyectos de intervención didáctica para desarrollar en los estudiantes aptitudes y actitudes positivas hacia las Ciencias Sociales" (Plan de Estudios, 2013, p.17).

\section{Definamos que es un Paradigma Investigativo}

La investigación Acción se enmarca dentro de una visión paradigmática, la cual es definida por (Guardián, 2007, p. 1) como "un sistema teórico dominante en la ciencia en cada período de su historia, que organiza y dirige la investigación científica en una cierta dirección, de igual forma permite el surgimiento de ciertas hipótesis e inhibe el desarrollo de otras, también centra la atención de quién investiga en determinados aspectos del tema de estudio o problema y oscurece otros". Al respecto (González, 2003, p. 125) define un paradigma como "una cosmovisión del mundo compartida por una comunidad científica; un modelo para situarse ante la realidad, interpretarla y darle solución a los problemas que en ella se presentan"

Por otra parte, (Alvarado y García, 2008, p. 190) consideran que un paradigma "es un cuerpo de creencias, presupuestos, reglas y procedimientos que definen cómo hay que hacer ciencia; son los modelos de acción para la búsqueda del conocimiento". (Martínez, 2007, p. 30) destaca que un paradigma incluye un conjunto de valores, creencias, metas, normas y lenguaje específico". También plantea que en la investigación educativa tanto investigadores, educadores y profesionales afines comparten un mismo paradigma o forma de interpretar la realidad educativa que se quiere investigar, para llegar a conjugar 
adecuadamente entre sí las teorías educativas, la metodología de investigación y la práctica educativa, con el fin de promover la calidad educativa.

Existen tres tipos de paradigmas investigativos. En el presente estudio se hace mención de tres visiones paradigmáticas, los cuales son el paradigma Positivista, el Interpretativo y el Socio Crítico. Cada una de estas visiones paradigmáticas de la investigación tiene características propias que difiere a cada uno de los otros. Por ejemplo: el paradigma Positivista hace uso de la estadística para llevarse a la práctica; el paradigma Interpretativo hace uso de técnicas cualitativas de investigación, para ello, en su proceso hace estudios sobre etnografía social o educativa; y el paradigma Sociocrítico tiene una característica que lo diferencia de los otros dos, y es que este paradigma una vez que identifica las problemáticas sociales o educativas les da solución, y para ello se auxilia de la Investigación Acción.

\section{Paradigma Sociocrítico y la Investigación Acción}

Cuando nos referimos a la Investigación Acción estamos haciendo alusión al paradigma Socio-Critico, el cual se enfoca en aspectos prácticos y observables. El mismo es definido por (Alvarado y García, 2008, p. 190) como un aporte sustancial a la teoría educativa y se destaca porque:

"El paradigma Socio-Crítico tiene como objetivo promover las transformaciones sociales, dando respuestas a problemas específicos presentes en el seno de las comunidades, pero con la participación de sus miembros. Se fundamenta en la crítica social con un severo sentido auto reflexivo, considera que el conocimiento se construye siempre por intereses de los grupos." Entre las características que más destaca ámbito educativo el autor citado en el párrafo anterior son:

- Adopción de una visión global y dialéctica de la realidad educativa.

- Aceptación de una visión democrática del conocimiento.

- Asunción de la teoría del conocimiento y de sus relaciones con la realidad y con la práctica.

También Kemmis \& Mctaggart (1992), aportan lo siguiente:

El paradigma Socio crítico utiliza el método investigación acción, es decir es una investigación colectiva, con el objeto de mejorar la racionalidad y la justicia de sus prácticas sociales o educativas. Este paradigma está relacionado permanentemente con este tipo de investigación (p.9).

Al respecto, Martínez (2007) plantea que el paradigma socio-crítico propone un método de investigación basado en la relación entre la teoría y la práctica, en el que se fomenta la investigación participativa. Su objetivo es formar a las personas para que desarrollen su capacidad de reflexión crítica y les permita analizar su propio contexto y realidad cotidiana, y tomen sus propias decisiones sobre las acciones que más les conviene realizar para hacer frente a sus limitaciones.

Las características fundamentales que señala Martínez (2007) en esta línea de investigación son las siguientes:

- El tema que es objeto de estudio surge de una comunidad de personas y en un contexto determinado.

- El objetivo principal de la investigación es conocer la situación de esa comunidad de sujetos y buscar alternativas de mejora.

- Se busca desarrollar en las personas procesos de reflexión sobre su propia situación, estimulando el desarrollo de la confianza en sí mismo, en sus capacidades y recursos.

- Pretende obtener resultados positivos para la situación estudiada.

\section{La Investigación Acción como herramienta para la formación docente}

De acuerdo con Latorre (2003) la Investigación Acción "se configura en cuatro momentos o fases: planificación, acción, observación y reflexión. El 
momento de la observación, la recogida y análisis de los datos de una manera sistemática y rigurosa, esto es lo que le otorga el rango de investigación" (p.21).

Por otra parte, Sagastizabal y Perlo (2002) consideran que "la investigación-acción, es una secuenciación de diagnósticos que intentan explicar la realidad y reorientar las acciones para lograr su cambio". (p. 6). También explican que este modelo de investigación "incorpora la acción y su posterior evaluación. Sus momentos de investigación-acción son los siguientes: Formulación del problema, recolección de datos, análisis de los datos, la acción, la evaluación". (p. 8).

Mckernan (1999), también hace referencia al procedimiento investigativo en la investigación acción, destacando que el diario es un documento personal, una técnica narrativa y registro de acontecimientos, pensamientos y sentimientos que tienen importancia para el autor. Como registro, es un compendio de datos que puede alertar al profesor para el desarrollo del pensamiento, los cambios en valores, el avance y la regresión para los que aprenden. El diario reúne los sentimientos y las creencias capturados en el momento en que han ocurrido o justo después, proporcionando así una "dimensión de estado de ánimo" a la acción humana.

La Investigación Acción es un método de investigación vital para las ciencias sociales e importante para los procesos educativos, en este sentido Anderson y Herr (2007), expresan que la Investigación Acción en el campo de la educación se utiliza principalmente como una forma de desarrollo profesional. De igual manera explican que, para que la investigación acción sea aceptada como metodología legítima, los investigadores tendrán que formular algunos criterios de validez. Esto no quiere decir que tengan que justificarse a través de los mismos criterios de la investigación tradicional, pero tendrán que convencer a los demás investigadores que este tipo de investigación es capaz de producir conocimientos importantes y válidos dentro de criterios congruentes con su metodología.
También Kemmis \& Mctaggart (1992), aportan puntos relevantes a la investigación acción al considerar que:

- La investigación acción se propone mejorar la educación mediante su cambio, y aprender a partir de las consecuencias de los cambios.

- La investigación acción es participativa: permite a las personas trabajar por la mejora de sus propias prácticas.

- La investigación acción crea comunidades autocríticas que participan y colaboran en todas las fases del proceso de investigación.

- La investigación acción es un proceso sistemático de aprendizaje en el que las personas actúan conscientemente a través de la inteligencia crítica que está orientada a dar forma a nuestra acción y a desarrollarla de tal modo que nuestra acción educativa sea comprendida.

\section{Etnografía educativa y la Investigación Acción}

De acuerdo con Goetz y LeCompte (1988), la etnografía es una forma de estudiar la vida humana, es una descripción o reconstrucción analítica de escenarios y grupos culturales intactos. El investigador etnográfico comienza examinando grupos y procesos muy comunes; ello le permite apreciar los aspectos, tanto generales como de detalle, necesarios para dar credibilidad a su descripción. Las mismas explican que las estrategias etnográficas de investigación son empíricas y naturalistas, se recurre a la observación participante y no participante para obtener datos empíricos de los fenómenos reales, procurando que los investigadores eviten la manipulación intencional de las variables del estudio.

Según Goetz y LeCompte (1988), la etnografía educativa abarca el conjunto de literatura, resultados, conclusiones, interpretaciones y teorías derivadas de los estudios de campos sobre la enseñanza escolar y otros procesos educativos. El objeto de la etnografía educativa es aportar valiosos datos descriptivos de los contextos, actividades y creencias de los participantes en los escenarios educativos. 
En la presente investigación, se hizo uso de la etnografía como estrategia de investigación la cual permitió estudiar las problemáticas del fenómeno en estudio, de manera precisa y exacta.

En este sentido Rossi y O’Higgins (1981) considera que los etnógrafos intentan describir aquellos aspectos de la conducta que son más o menos comunes y habituales al grupo o a un significativo segmento de él. También señala que la etnografía hace uso de los métodos de campo, entrevista con los informantes y notas de campos.

La etnografía es una estrategia de investigación aplicable en los espacios educativos tales como en las Ciencias Sociales y la Antropología, y por tal razón es pertinente definir el rol de la etnografía en la educación.

De acuerdo con Kemmis \& Mctaggart (1992) la Investigación Acción es una forma de indagación introspectiva colectiva emprendida por participantes en situaciones sociales con la intención de mejorar la racionalidad y la justicia de sus prácticas sociales o educativas. La introspección la realizan los docentes en funciones, y para ello toman sus aulas de clases como laboratorio para hacer innovaciones didácticas. Estas innovaciones las llevan a cabo una vez que la universidad les da las herramientas metodológicas y didácticas para hacer intervenciones didácticas para mejorar los procesos de aprendizaje de sus estudiantes en las disciplinas que imparten en el subsistema de educación media.

En la Investigación Acción Participativa como método de investigación, se rompe la dicotomía sujeto-objeto de investigación, y se genera así una unidad o un equipo de investigación integrado, por un lado, por expertos investigadores, quienes cumplen el rol de facilitadores o agentes del cambio; por otro, por la comunidad o grupos donde se realizan la investigación, quienes serán los propios gestores del proyecto investigativo $\mathrm{y}$, por ende, protagonistas de la transformación de su propia realidad y constructores de su proyecto de vida (Bernal, 2004).

Con base en los aspectos planteados por Rossi \& O’Higgins (1981); Goetz y LeCompte(1988); Kemmis \& Mctaggart (1992); Mckernan (1999); Sagastizabal \& Perlo (2002); Latorre (2003); Bernal, (2004); Anderson \& Herr (2007) \& Martínez (2007) en la asignatura Didáctica de las Ciencias Sociales ${ }^{1}$ (DCS) de la Carrera Ciencias Sociales se diseñan proyectos de intervención didáctica dirigidos a la mejora de los procesos de aprendizaje y enseñanza de los contenidos de Historia (de Nicaragua, América y Universal), Geografía (de Nicaragua, América y Universal); Economía; Filosofía, Sociología y Convivencia y Civismo. Estos proyectos se llevan a la práctica a través de la realización de intervenciones didácticas en las disciplinas antes mencionadas en Institutos de Educación Secundaria, esto con la intención de que los profesionales y graduados de esta carrera sean los agentes de cambio que se insertarán en el subsistema de educación secundaria.

Se puede afirmar que a través de la ejecución de este tipo de investigaciones en la carrera se logra ir trabajando en función de la misión de nuestra Facultad, la cual es "formar profesionales y técnicos en los campos de la educación e idiomas, con un pensamiento autónomo y convaloresmorales, éticosyunaactitudcrítica, reflexiva, emprendedora e innovadora, con las competencias para un auto aprendizaje continuo y permanente, en función de aportar al desarrollo equitativo y sostenible de la nación”( Plan Operativo de la Facultad, 2016)

A través de la realización, ejecución consciente y una actitud crítica, reflexiva e innovadora de los proyectos de intervención didáctica antes mencionados, se logrará formar a los profesionales y técnicos que requiere la nación para el desarrollo socioeconómico de los y las nicaragüenses. Pero, para realizar este tipo de trabajos investigativos es necesario que el futuro profesional

1 DCS significa Didáctica de las Ciencias Sociales 
de la educación y de las Ciencias Sociales tenga amor y vocación por la docencia, ya que de lo contario, se realizan trabajos de investigación como requisito de graduación sin ningún impacto en el buen desempeño de los docentes de educación secundaria.

A continuación, se hace mencionan de algunos de los trabajos realizados en la carrera en mención, donde se hace énfasis en la Investigación Acción, estas investigaciones fueron realizadas en los Institutos de
Educación Secundaria de nuestro país, a través de los cuales se ha incidido en la mejora de los procesos de aprendizaje de los contenidos de estudios sociales en los y las estudiantes de Educación Secundaria. Es importante mencionar que cada año en la carrera se realizan hasta 15 trabajos sobre la investigación acción y éstos se ejecutan en diferentes municipios de nuestro país, entre ellos, se hace mención a uno de los trabajos realizados por los estudiantes como formas de culminación de estudios, estos son los siguientes:

Tabla 1: Investigaciones realizadas en la carrera Ciencias Sociales basados en la investigación Acción.

Tema de la tesis $\begin{gathered}\text { Departamento/ } \\ \text { municipio }\end{gathered}$ Autores

Aplicación de estrategias de enseñanza-aprendizaje innovadoras para desarrollar la capacidad investigativa a través del contenido Rutas Turísticas de Nicaragua, disciplina Geografía de Nicaragua con los estudiantes del 10mo Grado "A" del Colegio San José, durante el I semestre 2008.

Aplicación de una unidad didáctica del contenido Globalización para desarrollar comprensión en los estudiantes del 10mo Grado del Centro Escolar Público “Andrés Castro” de Managua durante el I semestre, 2009.

Educación para el Consumo. Aplicación de una propuesta didáctica para generar comprensión en los estudiantes del 7mo Grado "E" del turno vespertino del Colegio Público La Salle de Diriamba, durante el I semestre 2010.

Una experiencia Didáctica para la enseñanza-aprendizaje del contenido I Guerra Mundial y el valor Tolerancia para desarrollar una cultura de paz en los y las estudiantes del 9no grado del turno matutino del Instituto Miguel De Cervantes Saavedra de Managua, durante el I semestre 2011.

Una propuesta metodológica con estrategias didácticas constructivistas para generar aprendizajes significativos en la disciplina Geografía de Nicaragua en los y las estudiantes del 7 mo grado del Colegio San Juan de Oriente, durante el II semestre 2012.

Aplicación de una simulación como estrategia de aprendizaje constructivista para generar comprensión en el contenido Asenso y Consolidación de la Dictadura Militar somocista en la disciplina Historia de Nicaragua, en los estudiantes del $7 \mathrm{mo}$ Grado del Colegio Rigoberto López Pérez, durante el II semestre 2013.

Intervención Didáctica para desarrollar la capacidad de relacionar e interpretar los procesos políticos, económicos y sociales en la disciplina Historia Universal en los contenidos Revolución Francesa y Revolución Popular Sandinista en los estudiantes de 9no Grado del Colegio Anexo Solidaridad, II semestre 2014.

Intervención Didáctica con Estrategias de Aprendizaje Innovadoras para generar Comprensión en la disciplina Historia a través del Contenido Lucha de Sandino en las y los estudiantes del $7 \mathrm{mo}$ grado D del Colegio Público Esquipulas del municipio de Managua, II semestre 2015.
Masatepe,

Masaya

Lea Ticay Chávez

Tipitapa,

Managua

María Lucrecia

Treminio Picado.

Diriamba, Carazo

Henry Vivas

Ligia Calero

Erika Navarrete

Managua,

Managua

San Juan de

Oriente, Masaya

Managua, Managua

Managua, Managua

Managua, Managua
Verónica Burgos

Juana Valle

Abner Navarrete

Mario Quintanilla Ivania Guevara

Sara Rivera

Evert Fonseca

Lea Marina Urbina

Johana Salazar

Mary Carmen Castillo

Adolfo Díaz

Manuel Mendoza

Ligia Arce 
Las investigaciones reflejadas en la tabla 1 han sido dirigidas o tutoradas por el autor del presente artículo producto de su formación como máster en Didáctica de las Ciencias Sociales y por la experiencia como formador de formadores en la Facultad de Educación e Idiomas de la UNAN- Managua. La experiencia ha dicho y lo han expresado los estudiantes (docentes en servicio en Educación Secundaria) que han realizado trabajos de tesis haciendo uso del paradigma Sociocrítico y la Investigación Acción, que han observado en sus estudiantes cambios de actitud hacia ellos y hacia las disciplinas sociales que ellos imparten. Por eso apostamos por la Investigación Acción como una herramienta didáctica para la formación de los docentes comprometidos con la educación y como verdaderos agentes de cambio en sus aulas de clases.

A continuación, se presenta una figura donde se muestra el número de estudiantes que se han graduado en la Carrera Ciencias Sociales en los últimos cinco (5) años. Estos han realizado sus tesis de grado haciendo uso del paradigma investigativo sociocrítico y la investigación acción.

Figura 1: Número de graduados en la carrera Ciencias Sociales

\section{Graduados carrera Ciencias Sociales 2011-2015}

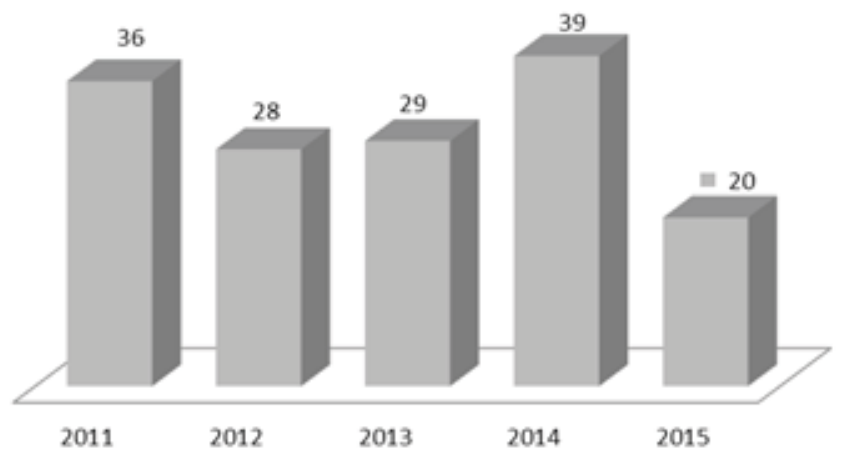

De acuerdo a los resultados reflejados en la figura 1, la carrera Ciencias Sociales tiene un promedio de 30 estudiantes que se gradúan por año. Respecto a los 36 estudiantes que se graduaron en el año 2011 se debe a que en ese grupo que realizó Seminario de Graduación, no todos pertenecen a esa cohorte. El incremento de estudiantes en ese año se debe a que en ese grupo se integraron estudiantes que estaban limpiando clases y no habían podido realizar su modalidad de graduación.

En cambio, en el 2012 y 2013 se mantiene el número de graduados, pero en la modalidad de graduación que se realizó en el 2014 incrementó debido al mismo fenómeno, algunos estudiantes quedan rezagados en el camino y realizan y presentan su trabajo de graduación hasta que han limpiado las asignaturas que contemplan el plan de estudios. Otros grupos incrementan porque el año anterior reprobaron la modalidad de graduación, por tanto, el año siguiente los grupos se agrandan y muchas veces los grupos se dividen por la cantidad de estudiantes. Por ejemplo, ahorita en el año 2016 hay inscritos para realizar Seminario de Graduación 46 estudiantes y ello se debe a que el año pasado muchos de los estudiantes que llegaron a $\mathrm{V}$ año debían clases hasta de 1er año de la carrera, otros debían clases de 3ro año y 4to año. Esta situación generó que para este II semestre 2016 el grupo se divida ya que un maestro no puede brindar atención y seguimiento a esta cantidad de estudiantes.

Tabla 2: Graduados por sexo y año en la carrera Ciencias Sociales 2011-2015

\begin{tabular}{|c|c|c|c|c|c|c|c|c|c|}
\hline \multicolumn{2}{|c|}{2011} & \multicolumn{2}{|c|}{2012} & \multicolumn{2}{|c|}{2013} & \multicolumn{2}{|c|}{2014} & \multicolumn{2}{|c|}{2015} \\
\hline $\mathbf{V}$ & $\mathbf{M}$ & $\mathbf{V}$ & $\mathbf{M}$ & $\mathbf{V}$ & $\mathbf{M}$ & $\mathbf{V}$ & $\mathbf{M}$ & $\mathbf{V}$ & $\mathbf{M}$ \\
\hline 15 & 21 & 9 & 19 & 6 & 23 & 12 & 27 & 6 & 14 \\
\hline \multicolumn{2}{|c|}{36} & \multicolumn{2}{|c|}{28} & \multicolumn{2}{|c|}{29} & \multicolumn{2}{|c|}{39} & \multicolumn{2}{|c|}{20} \\
\hline
\end{tabular}

V: varón; M:mujer

Fuente: Revisión de actas en el Sistema de Calificaciones, UNAN- Managua

Con relación a los datos expuestos en la tabla 2, donde se refleja que el 68\% de los graduados en los últimos 5 años son mujeres y solo el 32\% son varones. Quizá esto se debe a que la profesión docente es poco remunerada económicamente. Al respecto Lucio (2014), argumenta que para que la calidad docente mejore debe haber una serie de sinergias de los diferentes subsistemas, en la que todos unan fuerzas y esfuerzos por mejorar la calidad educativa, sin omitir los estímulos económicos a los profesores que son una parte sustancial para el buen ejercicio de la profesión docente. 


\section{CONCLUSIONES}

A partir de la revisión documental de las tesis de los graduados de la carrera Ciencias Sociales y de la investigación sobre la sistematización de las líneas de investigación implementadas en la carrera se concluye lo siguiente:

La Carrera Ciencias Sociales ha incidido de forma positiva en la formación docente haciendo uso de la Investigación Acción como método de investigación.

Los estudiantes que han realizado investigaciones basados en la Investigación Acción han mejorado de forma cualitativa su práctica docente. Este tipo de ejercicios les permite empoderarse de teorías educativas y de métodos de investigación cualitativas, facilitando de esta manera que los graduados de esta carrera puedan dirigir en sus centros educativos investigaciones que les permitan identificar los problemas de aprendizaje de sus estudiantes y darles solución, ya que concebirán el aula de clase como un espacio para experimentar una serie de estrategias didáctica innovadoras que permitan al estudiantado de Educación Secundaria aprender para la vida.

La carrera Ciencias Sociales a través de la preparación del profesorado ha incidido en la calidad de la educación, formando al personal docente que va a ejercer docencia en Educación Secundaria en el área de las Ciencias Sociales. De esta manera, nuestra facultad está concretizando su misión la cual es "formar profesionales y técnicos en los campos de la educación e idiomas, con un pensamiento autónomo y con valores morales, éticos y una actitud crítica, reflexiva, emprendedora e innovadora".

Cabe destacar que la UNAN- Managua está apoyando este tipo de trabajos investigativos, porque a través de la Dirección de Investigación de Grado está generando espacios para la innovación Pedagógica, para ello han creado una línea de innovación ${ }^{2}$ denominada

2 Ver III convocatoria (2016) de los fondos concursables de la UNAN- Managua en la página web de la institución "innovación socioeducativa" a través de la cual los estudiantes de pregrado pueden presentar sus innovaciones pedagógicas y si las mismas clasifican como tal, son financiadas con fondos del $6 \%$ institucional.

La investigación acción y el paradigma sociocrítico son la herramienta por excelencia para la formación del profesorado con visión, convicción y compromiso para gestar los cambios que necesitan los distintos subsistemas educativos de nuestro país. Pero para ello, hay que conocer en profundidad las bondades que tiene la utilización de este tipo de investigaciones en la formación del profesorado. Es importante destacar que la investigación acción no es exclusiva para las Ciencias Sociales, sino que es aplicable a todas las áreas del conocimiento, porque tiene la característica de concebir al aula de clase como un espacio o un laboratorio para la innovación pedagógica a través de la aplicación de estrategias didácticas innovadoras y que permitan al estudiante aprender para la vida.

Lucio en un artículo publicado en El Nuevo Diario (octubre 18, 2015) sobre la formación docente expresa que "un primer aspecto que da solidez, sentido y relevancia a las propuestas innovadoras de formación docente, es que las mismas posean una fundamentación filosófica clara, con amplio sentido democrático, participativo, de pertinencia y calidad de la educación.

En tanto esto se logre, más fácil será que la formación docente sea innovadora y prepare, con sentido de pertinencia y pertenencia, en función de realidad multicultural del país". Ante este planteamiento, la Investigación Acción y el paradigma sociocrítico da solución al docente que hace alusión este experto en temas de educación, porque la realización de una intervención didáctica innovadora permitirá la formación de un docente con las características y cualidades que hace alusión en este artículo publicado en un periódico nacional. 
En la carrera Ciencias Sociales, fundada en 1960, en promedio cada año se gradúan aproximadamente 20 estudiantes lo que indica que cada año se insertan al subsistema de Educación Básica y Media 20 profesionales de la educación con las competencias necesarias para ejercer la profesión docente de forma efectiva y eficiente.

Pero la experiencia nos dice, que para que estos profesionales pongan en práctica todas las herramientas didácticas y metodológicas desarrolladas durante sus estudios universitarios, el subsistema de Educación Básica y Media debe dar al profesorado mayor autonomía para que ejerzan la profesión con libertad y pongan en práctica ese potencial, creatividad, imaginación y curiosidad al poner en marcha las estrategias didácticas, las habilidades y destrezas desarrolladas en la Universidad.

\section{BIBLIOGRAFÍA}

Alvarado, L. y García, M. (2008, diciembre). Características más relevantes del paradigma sociocrítico. Revista universitaria de Investigación, 2 (9): 187-202.

Álvarez-Gayou, J. (2003). Cómo hacer una Investigación Cualitativa: Fundamentos y Metodologías. México: Paidós Educador

Anderson, G., \& Herr, K. (2007). El docenteinvestigador: la investigación-acción como una forma válida de generación de conocimientos. La investigación educativa: Una herramienta de conocimiento y de acción. Buenos Aires: Noveduc.

Bernal, A. (2006). Metodología de la Investigación. ( $2^{\text {da }}$ Edición) México: Pearson Educación.

Díaz, A., Mendoza, M. y Arce, L. (2015). Intervención Didáctica con Estrategias de Aprendizaje Innovadoras para generar Comprensión en la disciplina Historia a través del Contenido Lucha de Sandino en las y los estudiantes del 7 mo grado D del Colegio Público Esquipulas del municipio de Managua, II semestre 2015. Tesis de Licenciatura. Managua: UNAN- Managua.
Goetz, J. y LeCompte, M. (1988). Etnografía y diseño cualitativo en la investigación educativa. Madrid: Morata.

González, A. (2003, octubre-diciembre). Los paradigmas de investigación en las ciencias sociales. ISLAS, 45(138):125-135.

Gurdían-Fernández, A. (2007). El Paradigma Cualitativo en la Investigación Socio-Educativa. San José: PrintCenter.

Hernández, R. Fernández, C. y Baptista, P. (2006). Metodología de la investigación. (4ta ed.). México, D.F.: McGraw-Hill

Latorre, A. (2003). Investigación acción. España: Graó.

Lucio, R. (2014, 14 de marzo). Calidad del Docente, condición necesaria para mejorar la calidad educativa. El Nuevo Diario, p. 7A.

Martínez, R. (2007). La investigación en la práctica educativa: Guía metodológica de investigación para el diagnóstico y evaluación en los centros docentes. Madrid: CIDE.

McKernan, J. (1999). Investigación-acción y currículum: métodos y recursos para profesionales reflexivos. Estados Unidos: Morata.

Kemmis, S. y Mctaggart, R. (1992). Cómo Planificar la Investigación Acción. Barcelona: Laertes.

Orozco, J. (2001). Propuesta Didáctica para la enseñanza-aprendizaje del contenido los "30 años del periodo conservador" para generar en los alumnos un aprendizaje significativo en el I año de Secundaria del Instituto Autónomo Experimental México de Managua, durante el II semestre 2000. Trabajo Monográfico de Licenciatura. Managua: UNAN-Managua.

Orozco, J. (2006). Aplicación de Estrategias Metodológicas y su incidencia en el Aprendizaje Significativo del contenido Guerra Nacional en los estudiantes de 1er año de la Carrera Educación Infantil, durante el 1er semestre de 2006. Tesis de maestría. Managua: UNAN-MANAGUA.

Orozco, J. (2015). La didáctica de las Ciencias Sociales en la carrera Ciencias Sociales, impacto, en el desempeño de los docentes de Ciencias Sociales en 
Educación Secundaria. Trabajo de Fin de Máster en Educación e Intervención Social. Juigalpa, Chontales: FAREM-Chontales, UNAN-Managua. Orozco, J. (2016). Estrategias Didácticas y Aprendizaje de las Ciencias Sociales. Revista Cientifica de FAREM-Estelí. Medio Ambiente, Tecnología y Desarrollo Humano. (15), 65-80. Recuperado de: http://www.farem.unan.edu.ni/revistas/index.php/ RCientifica/article/view/242/222

Sagastizabal, M \& Perlo, C. (2002). La investigación- acción. (3 $3^{\text {ra }}$ ed.). Argentina: La Crujía.

Universidad Nacional Autónoma de Nicaragua, Managua (2011). Modelo Educativo, Normativa y Metodología para la Planificación Curricular. Managua: UNAN- Managua: Autor

Universidad Nacional Autónoma de Nicaragua, Managua (2013). Plan de Estudios Carrera Ciencias Sociales, Facultad de Educación e Idiomas. Managua: UNAN- Managua: Autor 